\title{
A terrain-following model of wave boundary layers
}

\author{
Jie $\mathrm{Yu}$ \\ Department of Civil Engineering, and School of Marine and Atmospheric Sciences Stony \\ Brook University, Stony Brook, New York 11794, USA
}

\begin{abstract}
Over a variable seabed, conventional boundary layer approximations are rendered to be inadequate because of the large variations in bed elevation in the direction of wave propagation. Applying the method of conformal transformation to map the flow domain with a corrugated boundary onto a uniform strip, we put forward a terrain-following modeling approach for Stokes boundary layer flows, accompanying the recent development of the exact Floquet theory of water waves over a generally periodic seabed. For a non-steep seabed profile, but not necessarily small undulation height compared with the water depth, we solve the vorticity equation to obtain the analytical solutions for the boundary layer velocities, bed shear stress and rate of viscous dissipation, explicitly showing the variations both across the boundary layer and along the bed. For a relatively steep bed profile, a remedy is proposed that allows the velocity profiles to be locally determined across the boundary layer avoiding solving the 2-D differential equation for the vorticity. The modeling methodology is presented here for a constant viscosity, including the case of constant eddy viscosity, but can be extended to the case of variable eddy viscosity to improve turbulence modeling. Keywords: Wave boundary layer, terrain-following, conformal map, periodic seabed
\end{abstract}

\section{Introduction}

Wave boundary layers at the seabed, also known as the Stokes boundary layers for oscillatory flows, are typically very thin compared with the water depth,

Preprint submitted to Journal of ${ }^{A} T_{E} X$ Templates

September 24, 2016

(C) 2016. This manuscript version is made available under the Elsevier user license http://www.elsevier.com/open-access/userlicense/1.0/ 
4 ranging from a few millimeters to centimeters depending on the bed and flow conditions (Mei 1989, Fredsøe \& Deigaard 1992, Nielsen 1992). The flow structure in this thin layer, however, is of great importance since it determines the bottom shear stress and near-bed turbulent intensity, hence having significant influence on sediment transport, physical, chemical and biological processes near the seabed. The wave boundary layer can also affect the vertical structure of a co-existing current in shallow coastal waters (Fredsøe \& Deigaard 1992). The fluctuating (in space and/or in time) bed stresses and pressure due to wave motion can induce, and interact with, groundwater flows (Mei 1989, Belibassakis 2012).

It is generally accepted in the literature that outside the wave boundary layer the potential flow field of wave propagation applies, which by itself is a mathematically challenging problem for generally varying topographies, even assuming the linear dynamics (Rhine \& Bretherton 1973, Athanassoulis \& Belibassakis 1999). Various approximate theories have been developed, manipulating the geometric constraints of seabed profiles, e.g. assuming a gentle slope, small bottom amplitude, or localized abrupt variation such as a step (see Athanassoulis \& Belibassakis 1999, and the references within). When the water wavelength, depth and the scale of bed variations are comparable, numerical or semi-numerical methods are generally sought. The computational costs can be significant even with the restriction to linear waves, depending on the complexity of seabed geometry. This has motivated studies to explore the methods for improvements. For instance, Athanassoulis \& Belibassakis (1999) developed the coupled-mode theory to amend the shortcoming in satisfying the bottom boundary conditions in numerical representations, thereby accelerating the numerical convergence. These authors also extended the coupled-mode method to treat oscillatory boundary layer effects over a generally variable bathymetry (Belibassakis \& Athanassoulis 2008). Porter \& Porter (2003) formulated a transfer matrix method for wave scattering by a patch of small-amplitude periodic bed, significantly reducing the computation. Most recently, Fokas \& Nachbin (2012) proposed a set of non-local equations in the propagation space for 
weakly nonlinear shallow water waves over a variable bottom using a numerical Schwarz-Christoffel mapping.

For a periodic seabed of arbitrary amplitude and shape, the recent advance in the Floquet theory of linear water waves (Yu \& Howard 2012) has provided a new and general approach that can be regarded as the definitive solution to this type of problems. What distinguishes this theory the most is not that it gives exact solutions but that for any frequency it gives a complete set of linear modes. For 2-D time harmonics motions over a horizontal flat seabed, it is well known that the general mathematical treatment provides a complete basis of solutions: Given a frequency, it consists of two oppositely directed propagating waves and two infinite families of evanescent waves (with rapidly exponentially growing wave amplitudes in opposite directions). This set of flat-bottom linear modes has, for many years, been the only known complete basis for water waves and played a significant role in various engineering and scientific applications. For a periodic seabed, the set of Floquet modes provided in Yu \& Howard (2012) is analogous to the above mentioned set of flat-bottom modes and in fact approaches the latter for the same given frequency when the bed undulation height is reduced to zero. Therefore, it becomes the second complete basis for water waves. It has been demonstrated that this set of Floquet modes can be used as a complete basis to construct solutions to boundary-value problems involving a finite extent of periodic bed, similar to the way that the flat-bottom basis is used (Howard \& Yu 2007, Yu \& Howard 2010, Yu \& Zheng 2012, Weidman et al. 2015).

This study is to provide the accompanying boundary layer formulation for linear waves over a periodic bed. Although the nonlinear effects become strong as the wave approaches the shoreline, complete and explicit linear solutions are still very valuable in engineering applications and studies of coastal environments and sustainability. In most practical situations, the wave boundary layer is thin for non-breaking waves and without a resulting rectified current. Even in the case of a turbulent condition, the turbulence is confined to the thin layer just above the bed (Jonsson 1966, Johns 1967, Fredsøe \& Deigaard 1992). The 
flow closely follows the seabed terrain, meaning that the Stokes boundary layer should be measured everywhere in the direction normal to the bed, not in the vertical $z$ direction. However, the conventional boundary layer approximation in the vertical $(x, z)$ plane assumes $\partial / \partial z \gg \partial / \partial x$. This becomes inappropriate when the seabed elevation varies at the scale comparable to the water depth. The conformal transformation developed in Yu \& Howard (2012) maps the flow domain over a periodic seabed onto a uniform strip with a flat bottom; see figure 1. Since the transformation preserves angles, a vertical distance just above the flat bottom in the mapped plane $(\xi, \eta)$ corresponds to a normal distance measured from the bed surface in the physical $(x, z)$ plane. Thus, a boundary layer approach formulated in the mapped plane is terrain-following, and the approximations would be made by comparing the derivatives normal and tangent to the bed surface, which respectively measure the variations across the boundary layer and along the bed.

In this study, we assume a constant mean water depth over the general periodic seabed. For many practical applications at scales of engineering interests, this assumption is appropriate since typical beach slopes are gentle (of a few degrees) and the mean water depths do not change significantly. In real coastal regions, a long stretch of periodic (or nearly periodic) seabed is uncommon, but patches of bed undulations of different shapes can occur over a long distance on a shoaling beach. The terrain-following boundary layer approach presented here can be applied locally, considering a finite stretch of periodic seabed. The corresponding potential wave field in such a finite domain can readily be obtained, following the procedure in Yu \& Zheng (2012). Here, for the simplicity and clear illustration, we shall only consider a free wave (a Floquet propagating mode) over an indefinitely long periodic bed. For long range propagation of waves, the attenuation of wave energy occurs due to the boundary layer effects. The mathematical tools exist in the literature to deal with long range wave propagation, in particular over a flat or mildly varying seabed (see Mei 1989 and the references within), and in principle can be adapted to analyze the coupling effects of wave boundary layer and wave amplitude attenuation in the conformally 
mapped plane. These applications are of scientific and engineering interest, but beyond the scope of the present work and will be explored elsewhere.

Real seabed topographies are in general two-dimensional, but approximately one-dimensional periodic bed features are common (Mei 1989, Komar 1998, Pietrzak, Kranenburg \& Abraham 1990, Elgar, Raubenheimer \& Herbers 2003). The theoretical approach presented here can be applied to obtain an accurate first order approximation in those situations. On the other hand, periodic seabeds are often used in laboratory to study wave-topography interactions. The theoretical predictions of the potential wave field and wave boundary layer over a periodic bed are desirable in planning and designing experiments, as well as analyzing data.

Whereas the fully nonlinear and terrain-following wave boundary layer dynamics is described by the vorticity equation in the mapped plane, cf. 3.10, the linearized equation for the Stokes boundary layer 3.13 is based on the assumption of small amplitude waves of the outer potential flows. Neither of these equations makes assumption on the steepness of seabed undulations, nor on the relative importance of the normal and tangential derivatives. They can be solved numerically if needed, matching with the outer potential wave field that can be provided by the Floquet theory (Yu \& Howard 2012) if linear waves are considered or by other wave theories deemed to be appropriate. However, for non-steep topographies (but not necessarily small undulation height compared with the water depth), we can further simply (3.13), obtaining explicit analytical solutions of the boundary layer velocity distribution and bed shear stress. This is the main focus of this study.

The rest of the paper is structured, as follows. In the Floquet theory of waves over a periodic seabed ( $\mathrm{Yu} \&$ Howard 2012) is summarized, where the conformal transformation is described. The terrain-following modeling of boundary layer flows is formulated in $\$ 3$. For a non-steep but large bed undulation compared with the water depth, the analytical solutions are given in $\$ 3.1$ Degradation of the solutions as the seabed becomes steep is discussed and, a simple remedy is proposed in $\$ 3.2$. Summarizing remarks follow in $\$ 4$. 


\section{The exact Floquet theory of waves over a periodic seabed}

Deferring the technical details to Yu \& Howard (2012), we here summarize the Floquet theory of water waves over a generally periodic seabed for the purpose of providing the potential flow field outside the boundary layer and introducing the conformal map that is needed in $\$ 3$ to formulate the terrainfollowing boundary layer approach.

\subsection{The conformal map}

Consider a layer of homogeneous fluid over a periodic seabed. Denoting the dimensional variables by the prime, in the vertical $\left(x^{\prime}, z^{\prime}\right)$ plane $z^{\prime}=0$ is the undisturbed free surface and the seabed is at $z^{\prime}=-h_{0}^{\prime}+h_{b}^{\prime}\left(x^{\prime}\right)$, where $h_{b}^{\prime}\left(x^{\prime}\right)$ has a spatial period $\lambda_{b e d}$ and zero average in $x^{\prime}$ so that $h_{0}^{\prime}$ represents the mean water depth. The bed elevation varies over a height of $\Delta h_{b}^{\prime}=h_{b, \max }^{\prime}-h_{b, \min }^{\prime}$. A wavenumber

$$
k_{B}=\pi / \lambda_{\text {bed }}
$$

is defined which corresponds to a wavelength $2 \lambda_{\text {bed }}$ and is used for normalization, as follows.

$$
x=k_{B} x^{\prime}, \quad z=k_{B} z^{\prime}, \quad h_{b}(x)=k_{B} h_{b}^{\prime}\left(x^{\prime}\right), \quad h_{0}=k_{B} h_{0}^{\prime} .
$$

It is clear that $\Delta h_{b} \equiv h_{b, \max }-h_{b, \min }=k_{B} \Delta h_{b}^{\prime}$ is a measure of the steepness of a seabed profile. The normalized seabed wavelength is $\pi$, which sets the intrinsic periodicity of the medium in which waves propagate. This is for the mathematical convenience to employ the Floquet theory, following the common practice. The conformal map between the $(x, z)$ and $(\xi, \eta)$ plane is given by ( $\mathrm{Yu}$ \& Howard 2012)

$$
\begin{aligned}
& x=\xi-h \sum_{j=1}^{\infty}\left(b_{j} \sin 2 j \xi-c_{j} \cos 2 j \xi\right) \cosh 2 j \eta / \sinh 2 j h \\
& z=\eta-h \sum_{j=1}^{\infty}\left(b_{j} \cos 2 j \xi+c_{j} \sin 2 j \xi\right) \sinh 2 j \eta / \sinh 2 j h .
\end{aligned}
$$


It transforms the flow domain $-h_{0}+h_{b}(x) \leq z \leq 0$ into a uniform strip $-h \leq$ $\eta \leq 0$; see figure 1 . The fluid depth $h$ in the mapped plane $(\xi, \eta)$ and the Fourier coefficients $b_{j}, c_{j}$ are implicitly determined according to

$$
-h_{0}+h_{b}(x)=-h+h \sum_{j=1}^{\infty}\left(b_{j} \cos 2 j \xi+c_{j} \sin 2 j \xi\right)
$$

where

$$
x=\xi-h \sum_{j=1}^{\infty}\left(b_{j} \sin 2 j \xi-c_{j} \cos 2 j \xi\right) \operatorname{coth}(2 j h)
$$

and

$$
h_{0}=h+h^{2} \sum_{j=1}^{\infty} j\left(b_{j}^{2}+c_{j}^{2}\right) \operatorname{coth}(2 j h) .
$$

The constraint 2.6 results from $h_{b}(x)$ having zero average in $x$. The map 2.3 is $\pi$-periodic in both $x$ and $\xi$, therefore preserves the intrinsic periodicity $\pi$ of the problem. The Cauchy-Riemann conditions, $x_{\xi}=z_{\eta}$ and $x_{\eta}=-z_{\xi}$, are readily verified from 2.3 . Under the transformation, the Cartesian grids $\xi=$ constant and $\eta=$ constant are mapped onto the orthogonal curvilinear grids in the $(x, z)$ plane, which are terrain-following near the seabed; see figure 11(c). An iteration method using FFT to compute the map parameters $b_{j}, c_{j}$ and $h$ (collectively denoted as $\mathcal{B}$ ) from $2.4-2.6$ is detailed in Yu \& Howard (2012), in which the convergence of the series is also discussed. From this point on, for a given profile $h_{b}(x)$ we consider $\mathcal{B}$ to be known.

\subsection{The Floquet solutions for time-harmonic flows}

In the mapped plane, the Laplace equation for the velocity potential $\phi$ is solved, exactly satisfying the conformally transformed boundary conditions at the free surface and the seabed. Using the normalization

$$
t=t^{\prime} \sqrt{g k_{B}}, \quad \phi=\phi^{\prime}\left(a \sqrt{g k_{B}} / k_{B}\right),
$$


where $a$ characterises the surface wave amplitude and $g$ is the acceleration gravity, we write the solution

$$
\phi=\mathrm{e}^{\mu \xi} \sum_{n=-\infty}^{\infty} D_{n} \mathrm{e}^{\mathrm{i} n \xi} \frac{\cosh [(n-\mathrm{i} \mu)(\eta+h)]}{\cosh [(n-\mathrm{i} \mu) h]} \mathrm{e}^{-\mathrm{i} \sigma t}+\text { c.c. },
$$

where the Floquet exponent $\mu$ is the generalized wavenumber for the given (normalized) frequency $\sigma=\omega / \sqrt{g k_{B}}$. Given a seabed, $\mu$ and $\sigma$ must satisfy the dispersion relationship $\Delta(\sigma, \mu ; \mathcal{B})=0$, where the function $\Delta$ is expressed as the determinant of a matrix which arises from satisfying the free surface boundary conditions. The Fourier coefficients $D_{n}$ are then given by the vector in the null space of the matrix. The entries of the matrix are explicit analytical functions of $\mu, \sigma$ and $\mathcal{B}$. Thus, the determinant $\Delta$ is an analytical function (similar to the Hill's infinite determinants) despite its complex appearance. The behavior of $\Delta$ and the convergence of the Fourier series in $(2.8)$ are discussed in $\mathrm{Yu} \&$ Howard (2012).

Given a frequency $\sigma$, a set of Floquet exponents (occurring in $\pm \mu$ pairs) can be found to satisfy $\Delta(\sigma, \mu ; \mathcal{B})=0$. Correspondingly, a set of solutions can be identified from 2.8): Two are referred to as the Floquet wave modes, which approach the flat-bottom propagating waves (in $\pm x$ directions) of the same frequency when the bed undulation height $\Delta h_{b} \rightarrow 0$ (or approach the flat-bottom standing waves if the frequency is in a 'forbidden band', i.e. Bragg resonant). The two infinite families of Floquet evanescent modes are given by a sequence of large real $\pm \mu$, having the wave amplitudes grow rapidly exponentially in the $+x$ or $-x$ direction. Each member of the families approaches a flat-bottom evanescent wave as $\Delta h_{b} \rightarrow 0$. For a particular problem involving a finite extent of periodic bed, the solution can be expressed as the superposition of this set of Floquet modes, with the proportionality coefficients being determined by satisfying the lateral boundary conditions in the $x$ direction (Yu \& Zheng 2012, Weidman et al. 2015).

Over a flat bed, waves with an arbitrary wavelength (i.e. the horizontal distance between two adjacent wave crests or troughs) are possible, since the 
length scale of the medium is indefinite. This is manifested by the one-toone relationship between the frequency $\omega$ and wavenumber $k$ (or $-k$ ). For a periodic seabed, however, the spatial periodicity of the bed sets an intrinsic length scale for the wave motions. Within the realm of linear dynamics, exactly spatially periodic waves with identical adjacent wave crests and troughs can only exist when the wavelength is an integer multiple of $\lambda_{b e d}$. These occur for the frequencies that correspond to $\pm \mu=\mathrm{i}(1-2 / m)$, giving the water wavelengths $\lambda=m \lambda_{\text {bed }}$, where the integer $m \geq 2$; see figure 2(a). For other frequencies, $\lambda \neq m \lambda_{\text {bed }}$, hence the two adjacent wave crests (or throughs) cannot be identical as they see different parts of the bed profile at any instance. As a result, the wave must modulate, slowly varying in space; see figure 2(b). These are the quasi-spatially periodic waves (similar to the Bloch waves in physics) that can freely propagate in an open domain of continuous periodic bed. Bragg resonant waves occur when $\lambda \simeq\left(2 / m^{\prime}\right) \lambda_{\text {bed }}, m^{\prime}=1,2, \cdots$, where the wave amplitudes vary in space exponentially but slowly since $\mu$ is real and small. (It is the slow variation that distinguishes the resonant waves from the evanescent waves.) Obviously, these resonant waves can only exist over a finite extent of periodic bed, where the lateral boundary conditions limit the spatial growth (or decay) of the wave amplitude. This was discussed in Yu \& Maas (2016) for internal waves in a two-layer fluid system, and is stressed here for free surface waves.

For time-harmonic wave motions over a large periodic seabed, since the spatial variation is not sinusoidal (even in the case of exactly spatially periodic waves), the propagation of wave is no longer seen as the simple translation of waveform in the space-time plane. The waveform changes as the wave propagates; see figure 2. Such modulations, which would have arisen from nonlinear effects in the case of wave propagation over a flat bottom, are here solely due to linear waves interacting with the seabed geometry. 
The parameters are

$$
\alpha=a k_{B}, \quad \delta=k_{B} \delta^{\prime},
$$

${ }_{232}$ where $\delta^{\prime}=\sqrt{2 \nu / \omega}$ is the usual definition of the Stokes layer thickness and $\nu$ is 233 the viscosity. Under the transformation, the no-slip boundary conditions at the 234

\section{A terrain-following boundary layer approach}

For 2-D incompressible fluid motions, the stream function $\psi(x, z, t)$ is so defined that the velocity vector

$$
(u, w)=\left(\psi_{z},-\psi_{x}\right)
$$

where the $x, z$ subscripts (as well as the $\xi, \eta$ subscripts below) are the standard notations for partial derivatives. The stream function is normalized by $a \sqrt{g k_{B}} / k_{B}$, same as for $\phi$. The vorticity $\boldsymbol{\nabla} \times \boldsymbol{u}=\nabla^{2} \psi \mathbf{j}$ is a pseudo vector perpendicular to the $(x, z)$ plane. In the $(\xi, \eta)$ plane, the exact vorticity equation

$$
\frac{\partial}{\partial t} \nabla^{2} \psi-\alpha \frac{\partial\left(\psi, J^{-1} \nabla^{2} \psi\right)}{\partial(\xi, \eta)}=\frac{1}{2} \delta^{2} \sigma \nabla^{2}\left(J^{-1} \nabla^{2} \psi\right),
$$

where the Laplacian operator

$$
\nabla^{2}=\frac{\partial^{2}}{\partial \xi^{2}}+\frac{\partial^{2}}{\partial \eta^{2}}
$$

and the Jacobian of transformation

$$
J(\xi, \eta)=x_{\xi}^{2}+x_{\eta}^{2} \neq 0
$$

seabed become

$$
\psi=\psi_{\eta}=0 \quad \text { at } \quad \eta=-h .
$$




$$
\frac{\partial}{\partial t}\left(\widetilde{\nabla}^{2} \widetilde{\psi}\right)-\alpha \frac{\partial\left(\widetilde{\psi}, J^{-1} \widetilde{\nabla}^{2} \widetilde{\psi}\right)}{\partial(\xi, \widetilde{\eta})}=\frac{1}{2} \sigma \widetilde{\nabla}^{2}\left(J^{-1} \widetilde{\nabla}^{2} \widetilde{\psi}\right) \quad \text { for } \quad \widetilde{\eta}>0
$$

where

$$
\widetilde{\nabla}^{2}=\delta^{2} \frac{\partial^{2}}{\partial \xi^{2}}+\frac{\partial^{2}}{\partial \widetilde{\eta}^{2}}
$$


is the distorted Laplacian operator. The boundary conditions in 3.6 become

$$
\widetilde{\psi}=\widetilde{\psi}_{\widetilde{\eta}}=0 \quad \text { at } \quad \widetilde{\eta}=0
$$

251

For linear waves $\alpha \ll 1$, we obtain

$$
\frac{\partial}{\partial t}\left(\widetilde{\nabla}^{2} \widetilde{\psi}\right)=\frac{1}{2} \sigma \widetilde{\nabla}^{2}\left(J^{-1} \widetilde{\nabla}^{2} \widetilde{\psi}\right) \quad \text { for } \quad \tilde{\eta}>0
$$

The effects of seabed are brought in via the Jacobian of transformation $J(\xi, \eta)$. This equation, together with the boundary conditions in 3.12 , describes the wave boundary layer flow following the bed surface. Given a periodic bed, it does not necessarily reduce to a 1-D differential equation (as in the conventional boundary layer approximations) since the bottom variation can be so rapid that $\delta \frac{\partial}{\partial \xi} \sim \frac{\partial}{\partial \eta}$.

\subsection{The analytical solutions for a non-steep periodic seabed}

For a non-steep seabed profile, the boundary layer flows can be sufficiently smooth so that $\delta \frac{\partial}{\partial \xi} \ll \frac{\partial}{\partial \eta}$. It then follows from 3.13 that

$$
\frac{\partial}{\partial t} \widetilde{\psi}_{\widetilde{\eta} \widetilde{\eta}}=\frac{1}{2} \sigma \frac{\partial^{2}}{\partial \widetilde{\eta}^{2}}\left(J^{-1} \widetilde{\psi}_{\widetilde{\eta} \widetilde{\eta}}\right) \text { for } \quad \widetilde{\eta}>0 .
$$

Close to the bed for $\widetilde{\eta}=O(1), J=J_{b}(\xi)+O(\delta \widetilde{\eta})$, where $\left.J_{b}(\xi) \equiv J\right|_{\eta=-h}$. To the lowest order approximation, we can further simplify (3.14) into

$$
\frac{\partial}{\partial t} \widetilde{\psi}_{\widetilde{\eta} \widetilde{\eta}}=\frac{1}{2} \sigma J_{b}^{-1} \widetilde{\psi}_{\widetilde{\eta} \widetilde{\eta} \widetilde{\eta}} \text { for } \quad \widetilde{\eta}>0 .
$$

Over a non-steep seabed, the Stokes boundary layer flow is essentially 1-D following the bed terrain with only a parametrical dependence on the along-bed variable $\xi$. This is similarly found in the study of oscillatory boundary layer flows over sand ripples (Hara \& Mei 1990). For time periodic motions, the solution that is bounded in $\widetilde{\eta}$ and satisfies the boundary conditions in 3.12 is found to be

$$
\widetilde{\psi}=C(\xi)\left[\widetilde{\eta}+\frac{1}{2}(1+\mathrm{i}) J_{b}^{-1 / 2}\left(\mathrm{e}^{-(1-\mathrm{i}) J_{b}^{1 / 2} \widetilde{\eta}}-1\right)\right] \mathrm{e}^{-\mathrm{i} \sigma t}+\text { c.c. },
$$


where $C(\xi)$ is to be determined by matching with the outer (potential) flow.

As is discussed in $\$ 2$, the linear potential flow above the seabed can be obtained using the appropriate superposition of the set of Floquet modes given by (2.8). For the illustration of completing the boundary layer solution (3.16), we suppose here that the potential flow in the inviscid core is given by a propagating Floquet wave mode. (For such a freely propagating wave to exist over an indefinite extent of periodic bed, the frequency is necessarily away from the forbidden bands.) For potential flows, $\phi$ and $\psi$ are conjugate harmonic functions, satisfying the Cauchy-Riemann conditions $\phi_{\xi}=\psi_{\eta}$ and $\phi_{\eta}=-\psi_{\xi}$. Thus, the velocities outside the boundary layer are given by

$$
U^{(o)}=J^{-1 / 2} \phi_{\xi}, \quad W^{(o)}=J^{-1 / 2} \phi_{\eta} .
$$

Comparing (3.17) and (3.9) at the outer edge of the boundary layer, we get

$$
\left.\phi_{\xi}\right|_{\eta \rightarrow-h}=\left.\widetilde{\psi}_{\widetilde{\eta}}\right|_{\widetilde{\eta} \rightarrow \infty},\left.\quad \phi_{\eta}\right|_{\eta \rightarrow-h}=-\left.\delta \widetilde{\psi}_{\xi}\right|_{\widetilde{\eta} \rightarrow \infty}
$$

It follows from 2.8 that at $\eta=-h+\delta \tilde{\eta}$

$$
\begin{aligned}
& \phi_{\xi}=\mathrm{e}^{\mu \xi} \sum_{n=-\infty}^{\infty} D_{n} \mathrm{e}^{\mathrm{i} n \xi} \frac{\mathrm{i}(n-\mathrm{i} \mu)}{\cosh [(n-\mathrm{i} \mu) h]} \mathrm{e}^{-\mathrm{i} \sigma t}+\text { c.c. }+O(\delta \widetilde{\eta}), \\
& \phi_{\eta}=\mathrm{e}^{\mu \xi} \sum_{n=-\infty}^{\infty} D_{n} \mathrm{e}^{\mathrm{i} n \xi} \frac{(n-\mathrm{i} \mu)^{2} \delta \widetilde{\eta}}{\cosh [(n-\mathrm{i} \mu) h]} \mathrm{e}^{-\mathrm{i} \sigma t}+\text { c.c. }+O(\delta \widetilde{\eta})^{3} .
\end{aligned}
$$

At the outer boundary, 3.16 gives

$$
\begin{aligned}
& \widetilde{\psi}_{\widetilde{\eta}} \rightarrow C \mathrm{e}^{-\mathrm{i} \sigma t}+\text { c.c. } \\
& \widetilde{\psi}_{\xi} \rightarrow\left[C^{\prime} \widetilde{\eta}-\frac{1}{2}(1+\mathrm{i}) J_{b}^{-1 / 2}\left(C^{\prime}-\frac{1}{2} C J_{b}^{\prime} / J_{b}\right)\right] \mathrm{e}^{-\mathrm{i} \sigma t}+\text { c.c. },
\end{aligned}
$$

where $C^{\prime}$ and $J_{b}^{\prime}$ are, respectively, the derivatives of $C(\xi)$ and $J_{b}(\xi)$. Within the thin boundary layer, $U$ and $W$ are essentially tangent and normal to the seabed, respectively. Matching $U$ at the outer boundary, cf. $(3.19 a)$ and $(3.20 b)$, 
yields

$$
C(\xi)=\mathrm{e}^{\mu \xi} \sum_{n=-\infty}^{\infty} D_{n} \mathrm{e}^{\mathrm{i} n \xi} \frac{\mathrm{i}(n-\mathrm{i} \mu)}{\cosh [(n-\mathrm{i} \mu) h]} .
$$

By the scaling of $\phi, C(\xi) \sim O(1)$. At the outer boundary,

$$
W^{(o)}-W^{(i)}=\delta \frac{1}{4}(1+\mathrm{i})\left(J_{b}^{\prime} J_{b}^{-2} C-2 J_{b}^{-1} C^{\prime}\right) \mathrm{e}^{-\mathrm{i} \sigma t}+\text { c.c. } \sim O(\delta) .
$$

Both tangential and normal velocities are matched up to the accuracy of $O(\delta)$ for non-steep seabeds, being consistent with the overall accuracy of the boundary layer approximation.

Writing $(U, W)=\frac{1}{2}\left(U_{m}, W_{m}\right) \mathrm{e}^{-\mathrm{i} \sigma t}+$ c.c., the amplitudes $\left|U_{m}\right|$ and $\left|W_{m}\right|$ vary considerably over a bed period, whereas the instantaneous velocities vary at the scale of water wavelength (or wave modulation length). In figures 3(a) and $3(\mathrm{~b}),\left|U_{m}\right|$ and $\left|W_{m}\right|$ are plotted varying in $z$ at different locations $\xi=\xi_{0}$ over a doubly sinusoidal bed, showing the structure of boundary layer velocities and matching with the outer velocities. Locally, the $U$ velocity profile across the boundary layer is qualitatively similar to that over a flat bed, but there is a significant variability in the velocity shear over a bed period $\pi$, obviously due to the large variation of bed elevation. Since the velocity gradients determine the bed shear stresses, these spatial variabilities in the boundary layer flow can have significant influence on the near-bed processes of sediments, chemical species and nutrients, as well as the submarine groundwater flows.

It must be mentioned that a vertical line $\xi=\xi_{0}$ in the mapped plane in general corresponds to a curve in the $(x, z)$ plane, which is perpendicular to the seabed and free surface. If the line $\xi=\xi_{0}$ is situated at a bed crest or trough (or other local elevation maximums or minimums), it corresponds to a vertical line $x=x_{0}$ in the $(x, z)$ plane; see figure $1(\mathrm{c})$. This is due to the fact that a conformal map preserves angles while stretching lengths. Therefore, $U$ and $W$ along $\xi=\xi_{0}$ are, respectively, the velocity components normal and tangent to the corresponding vertical curve in the physical plane: Close to $z=-h_{0}+h_{b}(x)$, 
$U$ is tangent and $W$ is normal to the seabed, whereas close to the free surface, they become the $u$ and $w$ velocities, respectively.

\subsection{Approximation for a steep periodic seabed}

As the seabed becomes steep, the derivative $J_{b}^{\prime}$ can be large at some locations, resulting in an appreciable discrepancy $W^{(o)}-W^{(i)}$, cf. 3.22 . To show an example, we take the doubly sinusoidal seabed in figure 3 and increase $h_{0}$ from $\pi / 4$ to $2 \pi / 5$, keeping the ratio $\Delta h_{b} / h_{0}=\frac{1}{2}$. This corresponds to shorten $\lambda_{b e d}$ by a factor of $5 / 8$, while fixing the corrugation height and mean water depth. The steepness of the bed profile is therefore increased from $\Delta h_{b}=\pi / 8$ to $\pi / 5$. For this steep bed profile, it is seen in figure 4 that $J_{b}^{-1}$, and $J_{b}^{\prime} J_{b}^{-2}$ in particular, vary rapidly and in large magnitude over a spatial period $\pi$. Consequently, the normal velocity $W$ cannot be matched properly at the outer boundary at some locations along the bed, even though the tangential velocity $U$ calculated using the solution (3.16) remains satisfactory; see in figure 5 the velocity profiles at $\xi_{0}=9.08,8.58$ and 7.88 .

This is of course due to the neglect of along-bed variations $\partial / \partial \xi$ in the vorticity equation (3.15), that are no longer small compared with the normal derivatives $\partial / \partial \eta$ across the Stokes layer due to the rapid changes in bed elevation. While the ultimate fix is to solve the fully 2-D linear equation $(3.13)$, a relatively simple remedy can be provided by solving (3.14). Although both are 1-D differential equations, 3.14 retains the full evaluation of $J(\xi, \eta)$, hence accounts for the 2-D effects of bed topography to a greater degree than 3.15) does. The equation (3.14), the seabed boundary conditions 3.12 and the matching conditions at the outer boundary form a 'two-point' boundary value problem which allows $U^{(i)}$ and $W^{(i)}$ to be locally determined along $\xi=\xi_{0}$. This improves the boundary layer velocity $W^{(i)}$, allowing a transition to match with the outer potential flow, but does not significantly affect the velocity $U^{(i)}$; see figure 5 A shooting method for solving a boundary layer equation (Cebeci \& Keller 1971) can be adapted to numerically integrate (3.14 along $\xi=\xi_{0}$. This is detailed in Appendix A. 


$$
n_{1}=h_{b, x} / \sqrt{1+h_{b, x}^{2}}, \quad n_{2}=-1 / \sqrt{1+h_{b, x}^{2}} .
$$

357 Upon using the map 2.3,

$$
n_{1}=J_{b}^{-1 / 2} x_{\eta}, \quad n_{2}=J_{b}^{-1 / 2} x_{\xi} \quad \text { at } \quad \eta=-h .
$$


Under the transformation,

$$
\psi_{z z}-\psi_{x x}=J_{b}^{-2}\left(x_{\xi}^{2}-x_{\eta}^{2}\right) \psi_{\eta \eta}, \quad \psi_{x z}=J_{b}^{-2} x_{\xi} x_{\eta} \psi_{\eta \eta} \quad \text { at } \quad \eta=-h .
$$

Substituting (3.27) and (3.28) into (3.24), we obtain the bed shear stress $\tau_{b} \equiv$ $\left.\tau_{s}\right|_{\eta=-h}$, i.e.

$$
\tau_{b}=\left.\frac{1}{2} \delta^{2} J_{b}^{-1} \psi_{\eta \eta}\right|_{\eta=-h}=\left.\frac{1}{2} \delta J_{b}^{-1} \widetilde{\psi}_{\widetilde{\eta} \tilde{\eta}}\right|_{\tilde{\eta}=0} .
$$

For a non-steep bed, using (3.16) we get

$$
\tau_{b}=\frac{1}{2}(1-\mathrm{i}) \delta J_{b}^{-1 / 2} C \mathrm{e}^{-\mathrm{i} \sigma t}+\text { c.c. }
$$

where the function $C$ is given in 3.21 . Writing $\tau_{b}=\frac{1}{2} \tau_{b m} \mathrm{e}^{-\mathrm{i} \sigma t}+$ c.c., the amplitude $\left|\tau_{b m}\right|$ fluctuates at the short-scale of topography, while the instantaneous $\tau_{b}$ varies over the scale of water wavelength (or wave modulation length, which is determined by the Floquet exponent $\mu$ for a given frequency $\sigma$ ). The shortwavelength fluctuation reflects the bed profile, but is not a copy or rescaled version of it; see figure 7. Recall that $U^{(o)}=J_{b}^{-1 / 2} C \mathrm{e}^{-\mathrm{i} \sigma t}+$ c.c. at $\eta=-h$. For a constant viscosity and simple time-harmonic motions, the phase shift of $\pi / 4$ (in time) remains between the bed shear stress and the outer velocity, same as in the case of a flat bed. Some mathematical details in deriving (3.28) and 3.29 are given in Appendix B.

Normalized by $\rho a^{2}\left(g k_{B}\right)^{3 / 2}$, the rate of viscous dissipation per volume in the fluid is

$$
\begin{aligned}
\dot{d} & =\frac{1}{2} \delta^{2}\left[2\left(u_{x}\right)^{2}+2\left(w_{z}\right)^{2}+\left(u_{z}+w_{x}\right)^{2}\right] \\
& =\frac{1}{2} \delta^{2}\left[4\left(\psi_{x z}\right)^{2}+\left(\psi_{z z}-\psi_{x x}\right)^{2}\right] .
\end{aligned}
$$

34 Close to the seabed at $\eta=-h+\sigma \widetilde{\eta}$,

$$
\psi_{x z} \simeq \delta^{-1} J_{b}^{-2} x_{\eta} x_{\xi} \widetilde{\psi}_{\widetilde{\eta} \tilde{\eta}}+O(1)
$$




$$
\psi_{z z}-\psi_{x x} \simeq \delta^{-1} J_{b}^{-2}\left(x_{\xi}^{2}-x_{\eta}^{2}\right) \widetilde{\psi}_{\tilde{\eta} \widetilde{\eta}}+O(1)
$$

375 To the lowest order approximation, the rate of viscous dissipation inside the boundary layer and over a bed period $0<\xi<\pi$ is

$$
\dot{D}=\int_{0}^{\pi} \int_{-h}^{-h+\delta \widetilde{\eta}_{\infty}} \dot{d} J \mathrm{~d} \xi \mathrm{d} \eta \simeq \frac{1}{2} \delta \int_{0}^{\pi} \int_{0}^{\widetilde{\eta}_{\infty}} J_{b}^{-1}\left(\widetilde{\psi}_{\widetilde{\eta} \widetilde{\eta}}\right)^{2} \mathrm{~d} \xi \mathrm{d} \widetilde{\eta}
$$

where $\widetilde{\eta}_{\infty}$ is the outer edge of the Stokes boundary layer that can easily be determined by examining the matching of velocities. For a non-steep seabed, using 3.16 we obtain the rate of dissipation averaged over a wave period

$$
\bar{D} \simeq \delta \int_{0}^{\pi} C C^{*} J_{b}^{-1 / 2}\left(1-\mathrm{e}^{-2 J_{b}^{1 / 2} \widetilde{\eta}_{\infty}}\right) \mathrm{d} \xi
$$

The integration above can be evaluated fairly easily, e.g. using Matlab.

\section{Concluding remarks}

For 2-D boundary layer flows under the wave motions over a generally periodic seabed, we have formulated a terrain-following modeling approach. Applying the conformal transformation developed in Yu \& Howard (2012), the Cartesian grids in the mapped plane correspond to the orthogonal curvilinear grids in the physical plane, which are terrain-following near the seabed. This allows the vorticity equation to be solved to advantage in the Cartesian coordinates in the mapped plane, obtaining the description of flow dynamics following the boundary terrain. For linear dynamics, the potential flow field above the periodic seabed is provided by the Floquet theory of water waves in Yu \& Howard (2012).

For a non-steep periodic seabed (but not necessarily small undulation height compared with the water depth), the linearized vorticity equation in the mapped plane is formally similar to that of the Stokes boundary layer over a flat bed, with only a parametric dependence on the along-bed variable. The analytical solutions are attainable, including the boundary layer velocity profiles, the bed 
shear stress and the rate of viscous dissipation. For a relatively steep seabed, the 1-D differential equation (3.14 can account for the 2-D effects of bed to a greater degree since it retains the full evaluation of the Jacobian of transformation. Thus, the velocity profiles across the Stokes layer can still be locally determined. For still steeper bed profiles, the fully 2-D differential equation 3.13 is necessary to obtain the Stokes boundary layer flow.

Although we have used the molecular viscosity when presenting the examples, a constant eddy viscosity for turbulent flows equally applies. To improve turbulence modeling, the terrain-following modeling methodology developed in this study can be extended to the case of spatially varying eddy viscosity. The conformal transformation of the vorticity equation may involve some tedious algebra, but is straightforward. The coefficients of the vorticity equation will be more complicated, combining the Jacobian of the transformation (the effects of bed geometry) and the functions describing the spatial variations of turbulence intensity.

The nonlinear dynamics of wave boundary layer flows can be studied in the mapped plane, in principle following the standard approaches in the literature (e.g. Hara \& Mei 1990, Yu \& Mei 2000). For a periodic bed, the Floquet theory of water waves ( $\mathrm{Yu} \&$ Howard 2012) is yet to be extended to include the surface wave nonlinearity, though other existing theories may be applied to provide the potential flow above the seabed (e.g. Fokas \& Nachbin 2012). Of particular interest is the spatial variability of the induced steady streaming and mass transport in the wave boundary layer, due to the non-uniformity of viscous resistance along the variable seabed, and the implications for near-bed processes. In a recent study of viscous flows driven by vibrating sawtooth channel walls (Yu 2014), the method of conformal mapping presented here has been shown to have a superior capability in modeling nonlinear boundary layer flows.

For non-periodic seabeds, the terrain-following modeling methodology presented here should in principle be applicable, provided that a conformal map is attainable, either analytically or numerically, and most importantly the potential flow field of waves over such a seabed is available. Of course, the mathe- 
matical explicitness is likely to be lost in such general cases.

The attention in this study is on large scale seabed topographies of wavelength comparable to, or greater than, the water wavelength, which cause wave scattering and modulation. For small scale bed ripples of wavelength comparable to the water wave amplitude under the essentially oscillatory flows (see the reviews in Blondeaux 2001, Charru, Andreotti \& Claudin 2013, Seminara 1998), the method of conformal mapping has been applied to study the boundary layer flows. In such studies, the known transformation functions are employed, limiting the ripple profiles to certain shapes, e.g. Hara \& Mei (1990). The method of constructing conformation transformation functions for arbitrary periodic shapes, as presented here, can be modified so that the mapping is more confined to the bed and less effective in the inviscid core. Using such a map, we can study the Stokes boundary layer and wave induced steady streaming over a rippled bed of large amplitude and complex form, e.g. following the analyses in Hara \& Mei (1990).

\section{Acknowledgements}

The author thanks L. R. M. Maas for valuable discussions and comments. The author would also like to express her gratitude to three anonymous reviewers for their careful reading the paper and constructive comments that have led to the improvement of the paper. Support to JY by US National Science Foundation (Grant CBET-0845957) during the period when the materials in this work were developed, is gratefully acknowledged.

\section{Appendix A. A shooting method for solving (3.14)}

Integrating 3.14 twice, we obtain

$$
\frac{\partial^{2} \widetilde{\psi}}{\partial \widetilde{\eta}^{2}}=-2 \mathrm{i} J(\xi, \eta)\left[\widetilde{\psi}-C_{2}(\xi) \widetilde{\eta}-C_{1}(\xi)\right]
$$


In view of the matching conditions in 3.18 , as well as 3.19 , we set

$$
\widetilde{\psi}=C(\xi) \widetilde{\eta}+G(\xi, \widetilde{\eta})
$$

From (A.1), 3.12) and (3.18), the equations for $G$ are obtained, i.e.

$$
\begin{aligned}
\frac{\partial^{2} G}{\partial \widetilde{\eta}^{2}} & =-2 \mathrm{i} J\left[G+\left(C-C_{2}\right) \widetilde{\eta}-C_{1}\right], \\
G & =0, \quad \frac{\partial G}{\partial \widetilde{\eta}}=-C \quad \text { at } \quad \widetilde{\eta}=0, \\
G & =\frac{\partial G}{\widetilde{\eta}}=0 \quad \text { at } \quad \widetilde{\eta}=\widetilde{\eta}_{\infty},
\end{aligned}
$$

where $\widetilde{\eta}_{\infty}$ is some "sufficiently large" value that can be easily determined in the calculations. To get the normal velocity $W$, we need an equation for $\widetilde{\psi}_{\xi}$, hence $G_{\xi}$ in view of $(\mathrm{A} .2)$. Taking the derivative with respect to $\xi$, we obtain from A.3 and A.4,

$$
\begin{gathered}
\frac{\partial^{2} G_{\xi}}{\partial \widetilde{\eta}^{2}}=-2 \mathrm{i} J_{\xi}\left[G+\left(C-C_{2}\right) \widetilde{\eta}-C_{1}\right]-2 \mathrm{i} J\left[G_{\xi}+\left(C^{\prime}-C_{2}^{\prime}\right) \widetilde{\eta}-C_{1}^{\prime}\right] \\
G_{\xi}=0, \quad \frac{\partial G_{\xi}}{\partial \widetilde{\eta}}=-C^{\prime} \quad \text { at } \quad \widetilde{\eta}=0, \\
G_{\xi}=\frac{\partial G_{\xi}}{\partial \widetilde{\eta}}=0 \quad \text { at } \quad \widetilde{\eta}=\widetilde{\eta}_{\infty}
\end{gathered}
$$

where the prime denotes the derivative in $\xi$.

For a given position $\xi=\xi_{0}$, the problem is to find the local values $C_{1}\left(\xi_{0}\right)$, $C_{2}\left(\xi_{0}\right), C_{1}^{\prime}\left(\xi_{0}\right)$ and $C_{2}^{\prime}\left(\xi_{0}\right)$ such that the solutions of the (spatial) initial value problem A.3, A.4 a), A.5 and A.6a) satisfy the outer boundary conditions A.4 $b$ ) and $(\mathrm{A} .6 \mathrm{~b})$. In this study, we employ the shooting technique developed in (Cebeci \& Keller 1971), which combines the Newton's method for the search. Defining

$$
y_{1}=G, \quad y_{2}=\frac{\partial G}{\partial \widetilde{\eta}}, \quad y_{3}=G_{\xi}, \quad y_{4}=\frac{\partial G_{\xi}}{\partial \widetilde{\eta}},
$$


tions, i.e.

$$
\begin{aligned}
\frac{\mathrm{d} y_{1}}{\mathrm{~d} \widetilde{\eta}}= & y_{2}, \\
\frac{\mathrm{d} y_{2}}{\mathrm{~d} \widetilde{\eta}}= & -2 \mathrm{i} J\left[y_{1}+\left(C-C_{2}\right) \widetilde{\eta}-C_{1}\right], \\
\frac{\mathrm{d} y_{3}}{\mathrm{~d} \widetilde{\eta}}= & y_{4}, \\
\frac{\mathrm{d} y_{4}}{\mathrm{~d} \widetilde{\eta}}= & -2 \mathrm{i} J_{\xi}\left[y_{1}+\left(C-C_{2}\right) \widetilde{\eta}-C_{1}\right] \\
& -2 \mathrm{i} J\left[y_{3}+\left(C^{\prime}-C_{2}^{\prime}\right) \widetilde{\eta}-C_{1}^{\prime}\right] .
\end{aligned}
$$

467 The boundary conditions in A.4 and A.6 are replaced by

$$
\begin{array}{r}
y_{1}=0, \quad y_{2}=-C, \quad y_{3}=0, \quad y_{4}=-C^{\prime} \quad \text { at } \quad \widetilde{\eta}=0 \\
y_{1}=y_{2}=y_{3}=y_{4}=0 \quad \text { at } \quad \widetilde{\eta}=\widetilde{\eta}_{\infty} .
\end{array}
$$

${ }_{468}$ If we denote the solution to the initial value problem for a given location $\xi=\xi_{0}$ ${ }_{469}$ by $y_{i}\left(\widetilde{\eta} ; C_{1}, C_{2}, C_{1}^{\prime}, C_{2}^{\prime}\right), i=1,2,3,4$, we seek for $C_{1}\left(\xi_{0}\right), C_{2}\left(\xi_{0}\right), C_{1}^{\prime}\left(\xi_{0}\right)$ and ${ }_{470} \quad C_{2}^{\prime}\left(\xi_{0}\right)$ such that

$$
y_{i}\left(\widetilde{\eta}_{\infty} ; C_{1}, C_{2}, C_{1}^{\prime}, C_{2}^{\prime}\right)=0, \quad i=1,2,3,4
$$

${ }_{471}$ Applying Newton's method to the above equation, we obtain the iterations for 472 updating $C_{1}, C_{2}, C_{1}^{\prime}$ and $C_{2}^{\prime}$, i.e.

$$
\left[\begin{array}{l}
C_{1} \\
C_{2} \\
C_{1}^{\prime} \\
C_{2}^{\prime}
\end{array}\right]^{(j+1)}=\left[\begin{array}{l}
C_{1} \\
C_{2} \\
C_{1}^{\prime} \\
C_{2}^{\prime}
\end{array}\right]^{(j)}-\left[\begin{array}{llll}
\frac{\partial y_{1}}{\partial C_{1}} & \frac{\partial y_{1}}{\partial C_{2}} & \frac{\partial y_{1}}{\partial C_{1}^{\prime}} & \frac{\partial y_{1}}{\partial C_{2}^{\prime}} \\
\frac{\partial y_{2}}{\partial C_{1}} & \frac{\partial y_{2}}{\partial C_{2}} & \frac{\partial y_{2}}{\partial C_{1}^{\prime}} & \frac{\partial y_{2}}{\partial C_{2}^{\prime}} \\
\frac{\partial y_{3}}{\partial C_{1}} & \frac{\partial y_{3}}{\partial C_{2}} & \frac{\partial y_{3}}{\partial C_{1}^{\prime}} & \frac{\partial y_{3}}{\partial C_{2}^{\prime}} \\
\frac{\partial y_{4}}{\partial C_{1}} & \frac{\partial y_{4}}{\partial C_{2}} & \frac{\partial y_{4}}{\partial C_{1}^{\prime}} & \frac{\partial y_{4}}{\partial C_{2}^{\prime}}
\end{array}\right]^{-1}\left[\begin{array}{l}
y_{1} \\
y_{2} \\
y_{3} \\
y_{4}
\end{array}\right],
$$

${ }_{473}$ where $y_{i}$ and their derivatives with respect to $C_{1}, C_{2}, C_{1}^{\prime}$ and $C_{2}^{\prime}$ are evaluated ${ }_{474}$ at $\widetilde{\eta}_{\infty}$. To find these derivatives of $y_{i}$, we consider the variational equations for 


$$
q_{i}=\frac{\partial y_{i}}{\partial C_{1}}, q_{i+4}=\frac{\partial y_{i}}{\partial C_{2}}, q_{i+8}=\frac{\partial y_{i}}{\partial C_{1}^{\prime}}, q_{i+12}=\frac{\partial y_{i}}{\partial C_{2}^{\prime}}, \quad i=1,2,3,4
$$

476

478

A.8. Defining

$$
\begin{gathered}
\frac{\mathrm{d} q_{1}}{\mathrm{~d} \widetilde{\eta}}=q_{2}, \quad \frac{\mathrm{d} q_{2}}{\mathrm{~d} \widetilde{\eta}}=-2 \mathrm{i} J\left(q_{1}-1\right), \quad \frac{\mathrm{d} q_{3}}{\mathrm{~d} \widetilde{\eta}}=q_{4}, \\
\frac{\mathrm{d} q_{4}}{\mathrm{~d} \widetilde{\eta}}=-2 \mathrm{i} J_{\xi}\left(q_{1}-1\right)-2 \mathrm{i} J q_{3}, \quad \frac{\mathrm{d} q_{5}}{\mathrm{~d} \widetilde{\eta}}=q_{6}, \quad \frac{\mathrm{d} q_{6}}{\mathrm{~d} \widetilde{\eta}}=-2 \mathrm{i} J\left(q_{5}-\widetilde{\eta}\right), \\
\frac{\mathrm{d} q_{7}}{\mathrm{~d} \widetilde{\eta}}=q_{8}, \quad \frac{\mathrm{d} q_{8}}{\mathrm{~d} \widetilde{\eta}}=-2 \mathrm{i} J_{\xi}\left[q_{5}-\widetilde{\eta}\right]-2 \mathrm{i} J q_{7} \quad \frac{\mathrm{d} q_{9}}{\mathrm{~d} \widetilde{\eta}}=q_{10}, \\
\frac{\mathrm{d} q_{10}}{\mathrm{~d} \widetilde{\eta}}=-2 \mathrm{i} J q_{9}, \quad \frac{\mathrm{d} q_{11}}{\mathrm{~d} \widetilde{\eta}}=q_{12}, \quad \frac{\mathrm{d} q_{12}}{\mathrm{~d} \widetilde{\eta}}=-2 \mathrm{i} J_{\xi} q_{9}-2 \mathrm{i} J\left(q_{11}-1\right), \\
\frac{\mathrm{d} q_{13}}{\mathrm{~d} \widetilde{\eta}}=q_{14}, \quad \frac{\mathrm{d} q_{14}}{\mathrm{~d} \widetilde{\eta}}=-2 \mathrm{i} J q_{13}, \quad \frac{\mathrm{d} q_{15}}{\mathrm{~d} \widetilde{\eta}}=q_{16}, \\
\frac{\mathrm{d} q_{16}}{\mathrm{~d} \widetilde{\eta}}=-2 \mathrm{i} J_{\xi} q_{13}-2 \mathrm{i} J\left(q_{15}-\widetilde{\eta}\right) .
\end{gathered}
$$

From A.9, the initial conditions are obtained, i.e.

$$
q_{1}=q_{2}=\cdots=q_{16}=0 \quad \text { at } \quad \tilde{\eta}=0 .
$$

Once the initial value problem defined by (A.8, A.9a), A.13 and A.14 is solved, the second term on the right-hand-side of A.11 can be evaluated for $\widetilde{\eta}=\widetilde{\eta}_{\infty}$ and consequently the next approximation $\left[C_{1}, C_{2}, C_{1}^{\prime}, C_{2}^{\prime}\right]^{(j+1)}$ can be computed. The initial value problem can readily be solved, e.g. using the Matlab solver function ode 45 .

Upon finding the appropriate values $C_{1}, C_{2}, C_{1}^{\prime}, C_{2}^{\prime}$ at $\xi=\xi_{0}$, we integrate A.8 from the bed $\widetilde{\eta}=0$ to the outer boundary $\widetilde{\eta}=\widetilde{\eta}_{\infty}$, with the initial conditions in A.9. Recall that $y_{2} \equiv G_{\widetilde{\eta}}$ and $y_{3} \equiv G_{\xi}$. From A.2, $\widetilde{\psi}_{\widetilde{\eta}}=$ $C(\xi)+y_{2}$ and $\widetilde{\psi}_{\xi}=C^{\prime}(\xi) \widetilde{\eta}+y_{3}$ for $\xi=\xi_{0}$. The tangential and normal velocities, $U$ and $W$, immediately follow. The numerical results are included in figures 5 and 6, comparing with the analytical solutions given by 3.16 for a non-steep bed. 
Under the transformation

$$
\frac{\partial}{\partial x}=J^{-1}\left(x_{\xi} \frac{\partial}{\partial \xi}+x_{\eta} \frac{\partial}{\partial \eta}\right), \quad \frac{\partial}{\partial z}=J^{-1}\left(x_{\xi} \frac{\partial}{\partial \eta}-x_{\eta} \frac{\partial}{\partial \xi}\right) .
$$

492

$$
\begin{aligned}
& \psi_{x x}=J^{-1}\left(x_{\xi} \frac{\partial}{\partial \xi}+x_{\eta} \frac{\partial}{\partial \eta}\right)\left[J^{-1}\left(x_{\xi} \psi_{\xi}+x_{\eta} \psi_{\eta}\right)\right], \\
& \psi_{z z}=J^{-1}\left(x_{\xi} \frac{\partial}{\partial \eta}-x_{\eta} \frac{\partial}{\partial \xi}\right)\left[J^{-1}\left(x_{\xi} \psi_{\eta}-x_{\eta} \psi_{\xi}\right)\right], \\
& \psi_{x z}=J^{-1}\left(x_{\xi} \frac{\partial}{\partial \xi}+x_{\eta} \frac{\partial}{\partial \eta}\right)\left[J^{-1}\left(x_{\xi} \psi_{\eta}-x_{\eta} \psi_{\xi}\right)\right] .
\end{aligned}
$$

${ }_{493}$ At $\eta=-h, \psi=\psi_{\eta}=0$, hence $\psi_{\xi}=\psi_{\xi \xi}=\psi_{\xi \eta}=0$. After some algebra, we 494 obtain from (B.2) that at the seabed $\eta=-h$,

$$
\psi_{x x}=J_{b}^{-2} x_{\eta}^{2} \psi_{\eta \eta}, \quad \psi_{z z}=J_{b}^{-2} x_{\xi}^{2} \psi_{\eta \eta}, \quad \psi_{x z}=J_{b}^{-2} x_{\xi} x_{\eta} \psi_{\eta \eta} .
$$

495 These lead to the expressions in (3.28). Substituting (3.25) and (3.27) into 496

$$
\left.\tau_{s}\right|_{\eta=-h}=-2 \delta^{2} J_{b}^{-1} x_{\xi} x_{\eta} \psi_{x z}+\frac{1}{2} \delta^{2} J_{b}^{-1}\left(x_{\eta}^{2}-x_{\xi}^{2}\right)\left(\psi_{z z}-\psi_{x x}\right) .
$$

Using (B.3), we obtain

$$
\begin{aligned}
\left.\tau_{s}\right|_{\eta=-h} & =\delta^{2} J_{b}^{-3} \psi_{\eta \eta}\left[-2 x_{\xi}^{2} x_{\eta}^{2}-\frac{1}{2}\left(x_{\xi}^{2}-x_{\eta}^{2}\right)\right]^{2} \\
& =\frac{1}{2} \delta^{2} J_{b}^{-3} \psi_{\eta \eta}\left(x_{\xi}^{2}+x_{\eta}^{2}\right)^{2} .
\end{aligned}
$$

498 499

Noting that $J_{b}=x_{\xi}^{2}+x_{\eta}^{2}$ at $\eta=-h$, we obtain $\left.\tau_{s}\right|_{\eta=-h}=\frac{1}{2} \delta^{2} J_{b}^{-1} \psi_{\eta \eta}$. This is the right-hand-side of the bed shear stress $\tau_{b}$ in 3.29 . 


\section{References}

Athanassoulis, G. A. \& Belibassakis, K. A. 1999 A consistent coupledmode theory for the propagation of small-amplitude water waves over variable bathymetry regions. J. Fluid Mech., 389, 275-301.

Belibassakis, K. A. \& Athanassoulis, G. A. 2008 A coupled-mode approach to nonlinear waves in finite depth. Viscous bottom boundary-layer flow. Proc. 8th Intern. Conference on Hydrodynamics, ICHD 2008. Nantes, France.

Belibassakis, K. A. 2012 Water-wave induced groundwater pressure and flow in variable bathymetry regions and sandy beaches by an enhanced couplemode model.Ocean Eng. 47, 104-118.

Blondeaux, P. 2001 Mechanics of coastal forms. Annu. Rev. Fluid Mech., 33, 339-370.

Cebeci, T. \& Keller, H. B. 1971 Shooting and parallel shooting methods for solving the Falkner-Skan boundary layer equation. J. Comput. Phys. 7, 289-300.

Charru, F., Andreotti, B. \& Claudin, P. 2013 Sand ripples and dunes. Annu Rev Fluid Mech. 45, 469-493.

Elgar, S., Raubenheimer, B. \& Herbers, T. H. C. 2003 Bragg reflection of ocean waves from sandbars. Geophys. Rev. Lett., 30, 1016. doi:10.1029/2002GL016351.

FokAs, A. S. \& Nachbin, A. 2012 Water waves over a variable bottom: a non-local formulation and conformal mappings. J. Fluid Mech. 695, 288-309.

Fredsøe, J. \& DeigaArd, R. 1992 Mechanics of Coastal Sediment Transport. World Scientific.

Hara, T \& Mei, C. C. 1990 Oscillating flows over periodic ripples. J. Fluid Mech. 211, 183-209. 
Howard, L. N. \& YU, J. 2007 Normal modes of a rectangular tank with corrugated bottom. J. Fluid Mech., 593, 209-234.

Johns, B. 1967 The damping of gravity waves in shallow water by energy dissipation in a turbulent boundary layer. Tellus XX, 2, 330-337.

Jonsson, I. G.1966 Wave boundary layers and friction factors. Proc. 10th Conf. Coastal Eng. ASCE, 127-148.

Komar, P. D. 1998 Beach Processes and Sedimentation. (2nd edition) PrenticeHall, Saddle River, NJ.

MeI, C. C. 1989 The Applied Dynamics of Ocean Surface Waves. World Scientific.

Nielsen, P. 1992 Coastal Bottom Boundary Layers and Sediment Transport. World Scientific.

Pietrzak, J. D., Kranenburg, C. \& Abraham, G. 1990 Resonant internal waves in fluid flow. Nature, 344, 844-847.

Porter, R. \& Porter, D. 2003 Scattered and free waves over periodic beds. J. Fluid Mech. 483, 129-163.

Rhines, P. \& Bretherton, F. 1973 Topographic Rossby waves in a roughbottomed ocean. J. Fluid Mech. 61, 583-607.

SeminarA, G. 1998 Stability and morphodynamics. Meccanica, 33, 59-99.

Weidman, P. D., Herczynski, A. Yu, J. \& Howard, L. N. 2015 Experiments on standing waves in a rectangular tank with a corrugated bed. $J$. Fluid Mech., 777, 122-150.

YU, J. 2014 Fluid ratcheting by oscillating channel walls with sawteeth. J. Fluid Mech., 761, 305-328.

YU, J. \& Howard, L. N. 2010 On higher order Bragg resonance of water waves by bottom corrugations. J. Fluid Mech., 659, 484-504. 
${ }_{553}$ YU, J. \& Howard, L. N. 2012 Exact Floquet theory for waves over arbitrary ${ }_{554}$ periodic topographies. J. Fluid Mech. 712, 451-470.

${ }_{555}$ YU, J. \& MAas, L. R. M. 2016 Linear waves in two-layer fluids over periodic 556 bottoms. J. Fluid Mech. 794, 700-718.

${ }_{557} \mathrm{YU}$, J. \& MeI, C. C. 2000 Formation of sand bars under surface waves. $J$. ${ }_{558} \quad$ Fluid Mech., 416, 315-348.

${ }_{559}$ YU, J. \& Zheng, G. 2012 Exact solutions for wave propagation over a patch of large bottom corrugations. J. Fluid Mech., 713, 362-375. 
(a)

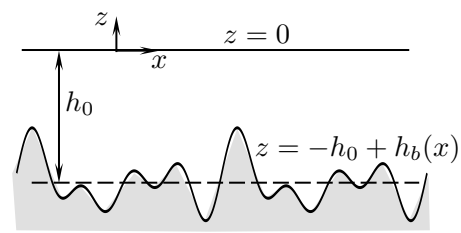

(b)

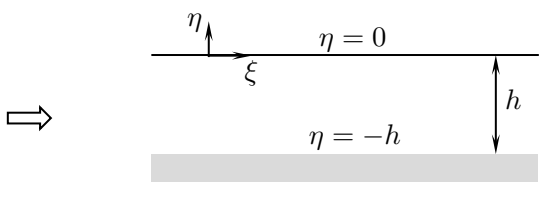

(c)

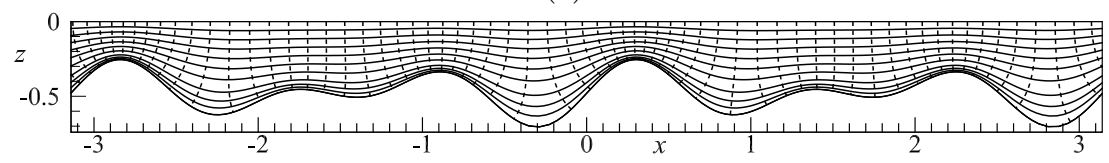

Figure 1: (a) A 2-D sketch of a homogeneous fluid layer over a periodic seabed, $-\infty<x<\infty$, $-h_{0}+h_{b}(x) \leq z \leq 0$. (b) The uniform strip of the flow domain upon conformal transformation, $-\infty<\xi<\infty,-h \leq \eta \leq 0$. (c) The orthogonal curvilinear grids in the $(x, z)$ plane that correspond to the Cartesian grids $\xi=$ constant and $\eta=$ constant, showing the terrainfollowing contours near the seabed. 
(a)

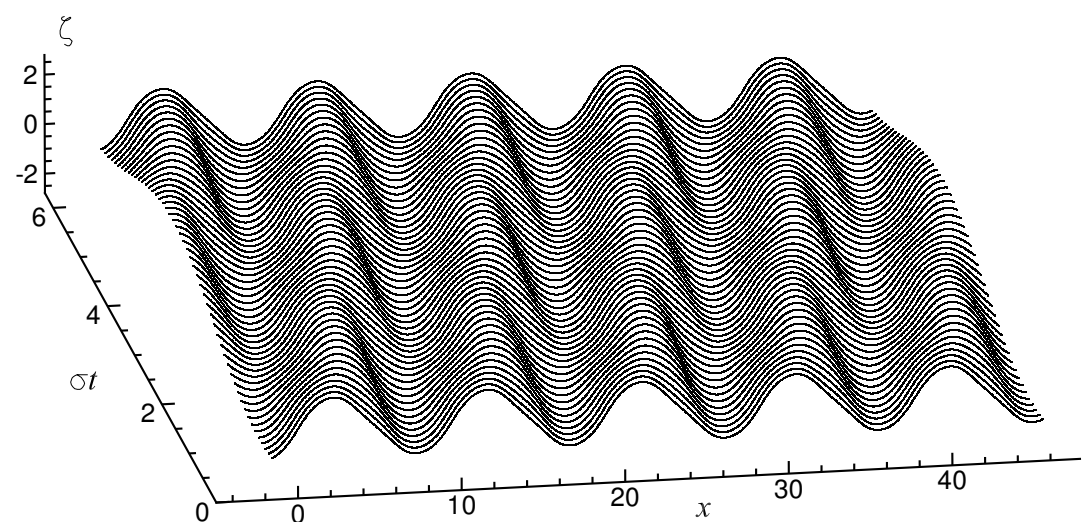

(b)

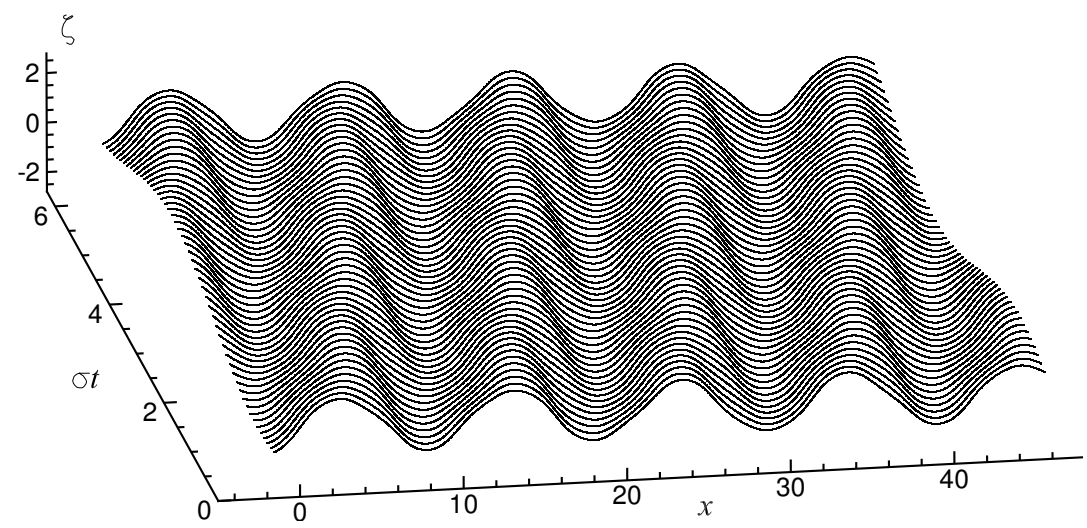

Figure 2: Surface elevations $\zeta(x, t)=-\left.\phi_{t}\right|_{z=0}$ for a wave propagating in the $+x$ direction over a simple sinusoidal bed. (a) A perfectly spatially periodic wave of wavelength (crest-to-crest distance) $3 \lambda_{\text {bed }}: \mu=-\mathrm{i} / 3$ and $\sigma=0.5977$. (b) A quasi-spatially periodic wave of wavelength $3.3 \lambda_{\text {bed }}: \mu=-\mathrm{i} 13 / 33$ and $\sigma=0.5488$. The seabed profile $h_{b}(x)=\frac{1}{2} \Delta h_{b} \cos (2 x), \Delta h_{b}=\frac{3}{4} h_{0}$ and $h_{0}=\pi / 3$. 
(a)

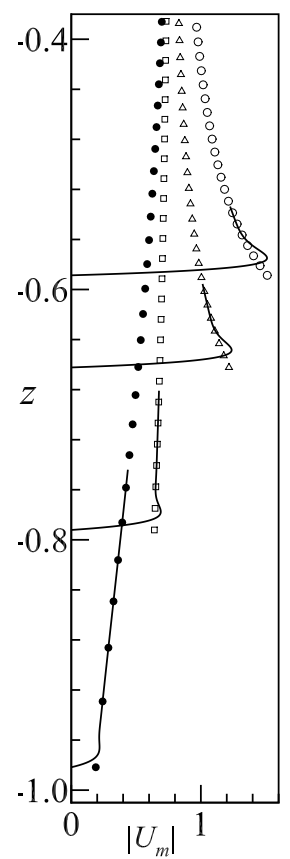

(b)

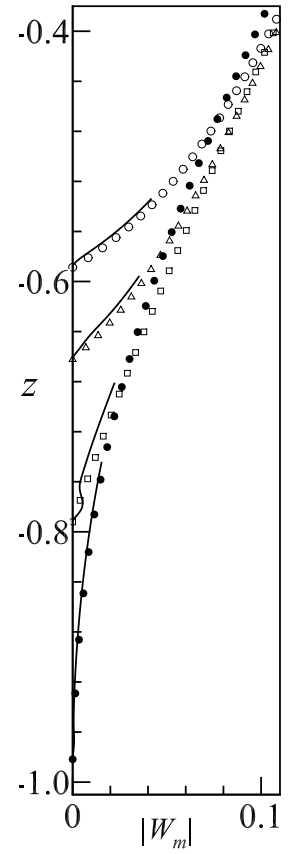

(c)

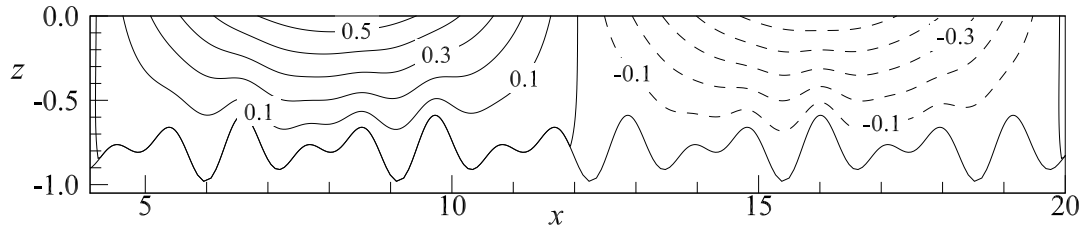

Figure 3: (a) The amplitude of $U$ along $\xi=\xi_{0}$ for a propagating wave of wavelength $5 \lambda_{b e d}$ over a doubly sinusoidal bed. (b) The amplitude of $W$. — , the boundary layer velocity given by (3.16). Symbols for the outer potential flow given by 2.8 ): $\bigcirc$, at $\xi_{0}=9.73$ (at a bed crest); $\bullet$, at $\xi_{0}=9.08$ (at a bed trough); $\triangle$, at $\xi_{0}=8.58 ; \square$, at $\xi_{0}=7.88$. (c) Contours of stream function $\psi$ at time $t=0$ over one wavelength in $x: \mu=-\mathrm{i} 3 / 5, \sigma=0.3388$ and $\delta=0.0064$. The bed profile $h_{b}(x)=\Delta h_{b}(\sin 4 x+\sin 6 x) / 3.8042, \Delta h_{b}=\frac{1}{2} h_{0}$ and $h_{0}=\pi / 4$. 


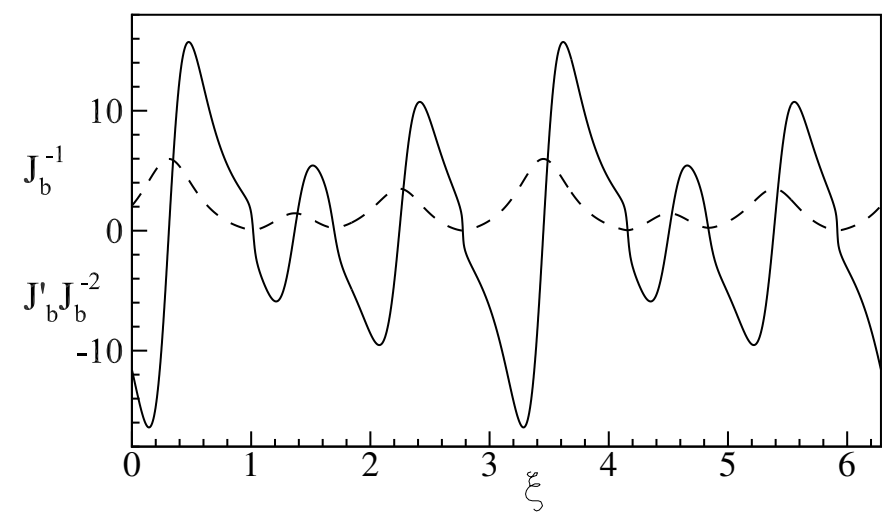

Figure 4: Graphs of $J_{b}^{-1}$ (dashed) and $J_{b}^{\prime} J_{b}^{-2}$ (solid) for $0<\xi<2 \pi$ for a steep doubly sinusoidal bed with $\Delta h_{b}=\frac{1}{2} h_{0}$ and $h_{0}=2 \pi / 5$.

(a)

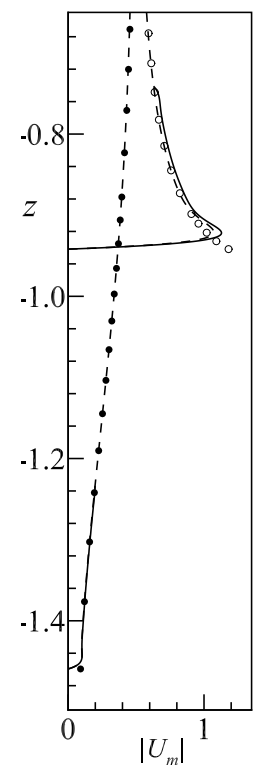

(b)

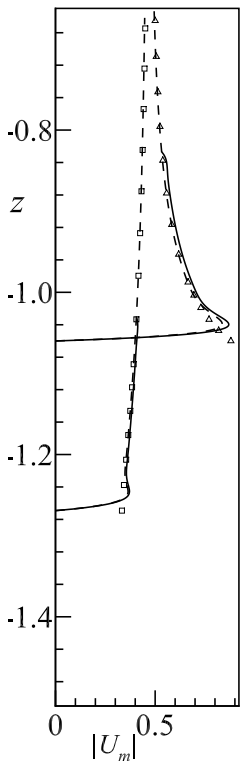

(c)

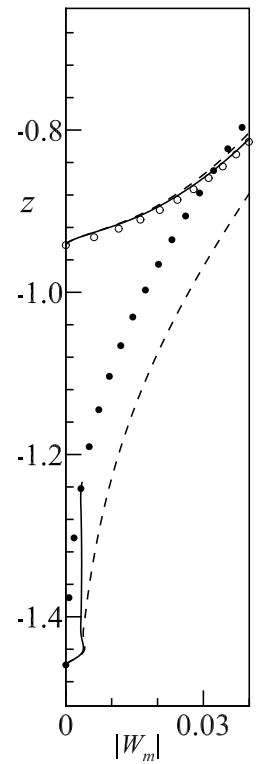

(d)

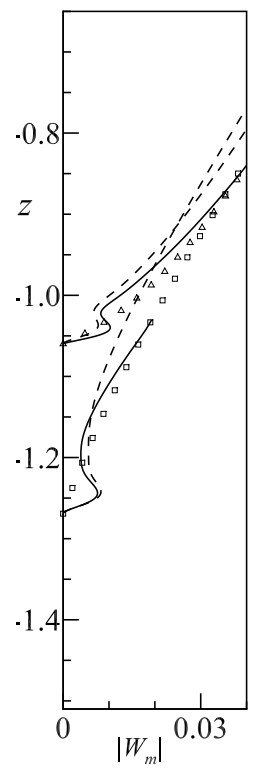

Figure 5: Comparison of the boundary layer velocities given by the solution 3.16 and by numerical integration of 3.14, for a wave of wavelength $8 \lambda_{\text {bed }}$ (keeping the same wavelengthdepth-ratio as in figure 3 over a steep doubly sinusoidal bed, $\Delta h_{b}=\frac{1}{2} h_{0}$ and $h_{0}=2 \pi / 5$. In (a) and (b), the amplitude $\left|U_{m}\right|$ along $\xi=\xi_{0}$; in (c) and (d), the amplitude $\left|W_{m}\right|$. ---, solution by 3.16; - - solution to 3.14. For the outer potential flow: $\bigcirc$, at $\xi_{0}=9.73 ; \bullet$, at $\xi_{0}=9.08 ; \triangle$, at $\xi_{0}=8.58 ; \square$, at $\xi_{0}=7.88$. The flow parameters, $\mu=-\mathrm{i} 3 / 4, \sigma=0.2646$, $\delta=0.01034$. 
(a)

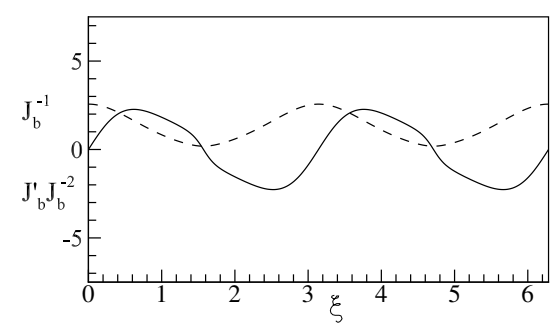

(b)

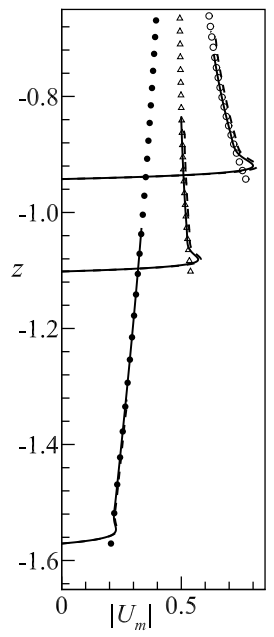

(c)

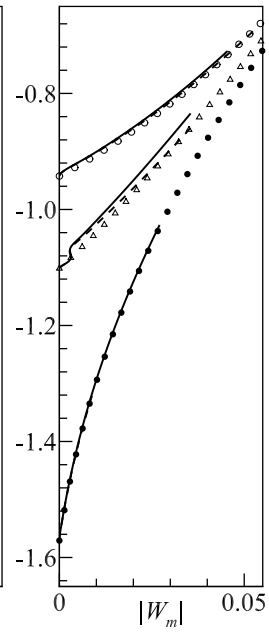

Figure 6: (a) Graphs of $J_{b}^{-1}$ (dashed) and $J_{b}^{\prime} J_{b}^{-2}$ (solid) for $0<\xi<2 \pi$ for a steep sinusoidal bed $h_{b}=\frac{1}{2} \Delta h_{b} \cos 2 x, \Delta h_{b}=\frac{1}{2} h_{0}$ and $h_{0}=2 \pi / 5$. (b) and (c) The amplitudes $\left|U_{m}\right|$ and $\left|W_{m}\right|$ along $\xi=\xi_{0}$ for a wave of wavelength $\lambda=8 \lambda_{\text {bed }}$. - - , solution by 3.16 ; ---, solution to 3.14. The outer potential flow: $\bigcirc$, at $\xi_{0}=0$ (bed crest); $\triangle$, at $\xi=\pi / 2$ (bed trough); $\bullet$ at $\xi=\pi / 4$. The flow parameters: $\mu=-\mathrm{i} 3 / 4, \sigma=0.2661$ and $\delta=0.0103$.

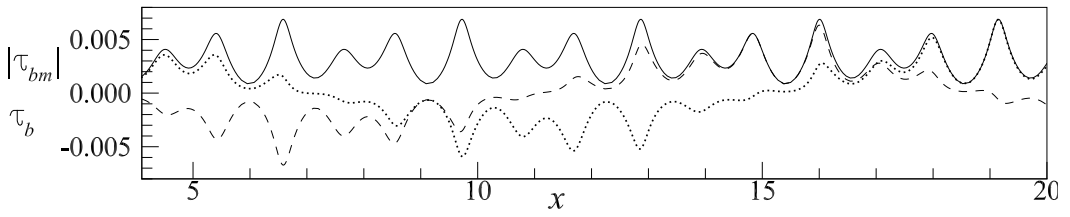

Figure 7: Spatial variations of bed shear stress over one water wavelength in $x$ along a doubly sinusoidal bed. - - the amplitude $\left|\tau_{b m}\right| ;---, \tau_{b}$ at $\sigma t=0 ; \cdots \cdots, \tau_{b}$ at $\sigma t=\pi / 2$. The conditions of the wave field and the seabed profile are the same as in figure 3 